\title{
Salivary Nitro-0xidative Stress and Matrix Metalloproteinase-9 in Chronic Kidney Disease with Periodontitis
}

\author{
Ciprian DALAI ${ }^{1}$, Alina Elena PÂRVU* ${ }^{2}$, Camelia DALAI ${ }^{1}$, Sandu Florin ALB ${ }^{3}$, Camelia ALB $^{4}$, Alina \\ ORMENIȘAN $^{5}$, Ciprian SILAGHI ${ }^{6}$, Ovidiu PÂRVU ${ }^{7}$ \\ ${ }^{1}$ Dental Medicine, Medicine and Pharmacy Faculty, 10 Piata 1 Decembrie, 410073 Oradea, Romania \\ ${ }^{2}$ Pathophysiology, ${ }^{3}$ Periodontology, ${ }^{4}$ Dental Propaedeutics, ${ }^{6}$ Medical Biochemistry, University of \\ Medicine and Pharmacy "Iuliu Hațieganu”, 8 Victor Babes Street, 400012 Cluj-Napoca, Romania \\ ${ }^{5}$ Oro-Maxilo Facial Surgery, University of Medicine and Pharmacy, $38 \mathrm{Gh}$. Marinescu Street, 540000 \\ Târgu Mureș, Romania \\ ${ }^{7}$ Department of Information Systems and Computing, Brunel University, Kingston Lane, UB8 3PH \\ Uxbridge, London, United Kingdom
}

Corresponding author, e-mail: parvualinaelena@yahoo.com

Bulletin UASVM Veterinary Medicine 71(2) / 2014,

Print ISSN 1843-5270; Electronic ISSN 1843-5378

DOI:10.15835/buasvmcn-vm: 10331

\begin{abstract}
Between chronic kidney disease and chronic periodontitis there is bilateral relationship. The aim of the study was to assess the salivary nitro-oxidative stress and matrix metallopeoteinase- 9 in chronic periodontitis and chronic kidney disease with chronic periodontitis. Eligible participants were categorized into three categories: healthy controls, chronic periodontitis and chronic kidney disease with chronic periodontitis. Periodontal status was assessed using probing depths, bleeding on probing, and clinical attachment level loss. The chronic kidney disease was diagnosed using the level of estimated glomerular filtration rate. From the saliva were assessed nitrooxidative stress biomarkers (total nitrites and nitrates, total oxidative status, total antioxidant reactivity, and oxidative stress index) and matrix metallopeoteinase-9. In both disease groups there was a significant increase in saliva nitro-oxidative stress due to an increased nitric oxide and reactive oxygen species formation $(\mathrm{p}<0.001)$ without a significant change of the antioxidants ( $p>0.05)$ and a concomitant increase of matrix metallopeoteinase-9 $(p<0.001)$. However, no statistically significant difference was observed $(p>0.05)$ when the intergroup comparison were made among the chronic periodontitis and chronic kidney disease with chronic periodontitis. Conclusions: Saliva nitro-oxidative stress markers and matrix metallopeoteinase-9 may be useful tests for pathogenetic and prognostic diagnosis in chronic periodontitis and chronic kidney disease with chronic periodontitis.
\end{abstract}

\section{Keywords}

chronic periodontitis, chronic kidney disease, saliva, nitric oxide, oxidative stress, matrix metaloproteinase-9

\section{INTRODUCTION}

Periodontal diseases are a group of chronic inflammatory diseases that affect the supporting tissues of the dentition. Periodontitis is one of the most frequent periodontal diseases. It is induced by the dental plaque bacteria in association with an abnormal host immune response to these microorganisms and their products in disease susceptible individuals (Machida et al., 2014; Craig et al., 2007). It is characterized by gingival bleeding, periodontal pocket formation, destruction of connective tissue attachment, and alveolar bone resorption. In periodontal lesions leukocytes produce reactive oxygen species (ROS), as an initial host defense against the bacterial pathogens (Tomofuji et al., 2011). ROS includes superoxide, 
hydroxyl, and nitric oxide radical species, as well as nonradical derivatives of oxygen. Excessive production of ROS disrupts the tissue oxidative/ antioxidative balance, and induces oxidative injury by damaging DNA, lipids, and proteins. Clinical studies have found that periodontitis patients have high blood levels of ROS compared to periodontally healthy subjects (Machida et al., 2014; Pârvu et al., 2013). Furthermore, it has been shown that periodontal treatment and some systemic treatments may decrease ROS in chronic periodontitis patients (Pârvu et al., 2014). Although it is still unclear whether oxidative status is the cause or result of periodontitis, it has been suggested that systemic oxidative stress can affect periodontitis progression, and may be a risk factor for systemic diseases, including diabetes mellitus, cardiovascular diseases, liver diseases, preterm low birth weight, hepatocellular carcinoma, and chronic kidney disease (CKD). Furthermore, mediators from systemic sources may amplify the local periodontal inflammatory cascade, but do not cause periodontitis by themselves (Ardalan et al., 2011; Wehmeyer et al., 2013; Pizzo et al., 2010; Graziani et al., 2010; Offenbacher, 1996).

More and more epidemiologic data support an association between periodontitis and chronic kidney disease (CKD) (Shaqman et al., 2010; Grubbs et al., 2011; Ismail et al., 2013). Compared to individuals with normal kidney function, individuals with reduced kidney function had higher prevalence of periodontitis (Ioannidou et al., 2013). CKD is a clinical syndrome induced by the progressive and irreversible loss of the glomerular filtration rate (Luczyszyn et al., 2012). CKD patients are characterized by a uremic milieu, which has been associated with immunosuppression (Ioannidou et al., 2011a; Ioannidou et al., 2014). Disturbances of the immune system in CKD involve both innate and adaptive immunity (Kato et al., 2008). Functional abnormalities of monocytes, neutrophils, and dendritic cells, impaired maturation of Th lymphocytes are directly linked to infection risk in this patient population (Anding et al., 2003; Ando et al., 2005; Carracedo et al., 2006). CKD may be associated with several oral manifestations, such as xerostomia, uremic stomatitis and periodontitis (Luczyszyn et al., 2012). In CKD patients salivary cytokines and secretion rates are significantly decreased (Thorman et al., 2010). Structural equation models exploring the bidirectional relationship of CKD and periodontitis suggested that CKD may have direct effect on moderate periodontitis, and that individuals with moderate periodontitis were more likely to have CKD (Ioannidou et al., 2011b; Pradeep et al., 2012; Dağ et al., 2010; Huang et al., 2014).

MMP-9 is a multidomain enzyme functioning in acute and chronic inflammatory diseases. It is mainly secreted by neutrophils although macrophages, T cells, mast cells, and odontoblasts can also express this enzyme. MMP-9 is essential for initiating the osteoclastic resorption process. In periodontitis, the expression of MMP-9 is found to be significantly higher than that in normal periodontal tissue (Wan et al., 2014).

The data on MMP-9 concentrations in CKD patients are scarce and contradictory and concern only adults (Musiał et al., 2010).

Local and systemic enhanced nitro-oxidative stress and MMP-9 in patients with CP were confirmed in saliva, GCF and plasma (Roberts et al., 2009; Roberts et al., 2010). A positive association between nitro-oxidative stress, MMP9 and chronic kidney disease (CKD) has been also reported. However, the effects of CKD association with periodontitis on saliva nitro-oxidative stress and MMP-9 are unknown. Therefore, the aim of our study was to evaluate the nitro-oxidative stress and the levels of MMP-9 in saliva samples from CKD patients with CP compared with CP nonCKD individuals.

\section{MATERIALS AND METHODS Materials}

Creatinine kit (AMEDA Labordiagnostik GmbH, Graz, Austria), and MMP-9 (gelatinase B) ELISA kits (R\&D Systems, Abingdon, UK), sulfanilamide (SULF), N-(1-Naphthyl) ethylendiamine dihydrochloride (NEDD), Vanadium (III) chloride $\left(\mathrm{VCl}_{3}\right)$ methanol, diethylether, xylenol orange [o-cresosulfonphthalein-3,3-bis (sodium methyliminodiacetate)], ortho dianisidine dihydrochloride (3-3'-dimethoxybenzidine), ferrous ammonium sulfate, hydrogen peroxide $\left(\mathrm{H}_{2} \mathrm{O}_{2}\right)$, sulfuric acid, hydrochloride acid, glycerol, and trolox (6-hydroxy-2,5,7,8-tetramethylchroman2-carboxylic acid) were purchased from Merck (Darmstadt, Germany) and Sigma-Aldrich (Taufkirchen Germany). Analytical grade chemicals were used exclusively. 


\section{Study design and population}

This was a prospective exploratory study that included 3 visits, randomized between January 2010 and December 2013. Test groups consisting of healthy controls (HC), patients with untreated chronic periodontitis (CP), or with CKD and CP (CKD-CP), were recruited from the Department of Periodontology, University of Medicine and Pharmacy Iuliu Hatieganu, Cluj Napoca (Romania) and the County Emergency Clinical Hospital Oradea, (Romania). Patient eligibility was evaluated during the first visit. At the first visit patients' medical and dental histories were obtained, and the patients underwent clinical and radiographic evaluation in order to diagnose periodontal disease. The CP group inclusion criteria were as follows: age, 30-60 years; at least 16 teeth present; at least 3 teeth in the same sextant; diagnosis of moderate to advanced chronic periodontitis; at least 2 sites with probing depths (PD) $\geq 5 \mathrm{~mm}$ together with bleeding on probing (BOP); $\geq 5-\mathrm{mm}$ clinical attachment level (CAL) loss; and radiographic evidence of alveolar bone height loss (4). At the second visit, blood samples were collected to assess renal function. The CKD criteria were defined according to clinical practice guidelines, using the level of estimated glomerular filtration rate (eGFR) (Redal-Baigorri et al., 2013; Levey et $a l ., 2014)$. Patients were excluded according to the following criteria: younger than 30 years and older than 70 years; receiving any pharmacological treatment within the 3 months before the beginning of the study; had already been treated for periodontal disease in the previous 6 months; clinically significant or unstable organic diseases other than CKD; compromised healing potential, such as connective tissue disorders or bone metabolic diseases; females who were pregnant or lactating, or who were of childbearing potential and not utilizing birth control or abstinence; cigarette smokers; active infectious diseases; and immunocompromised patients or those taking steroid medications. Finally, eligible participants (n $=168$ ) were categorized into 3 mutually exclusive categories ( $\mathrm{n}=56$ /group): HC, CP, and CKD with CP (CKD-CP). The selection for this study was randomized by age and gender matching. At the third visit saliva samples were collected to assess local nitro-oxidative stress and MMP-9 levels.

Every subject who fulfilled the inclusion criteria was provided with detailed information about the study and invited to participate. Written informed consent was obtained from all study participants before clinical periodontal examination and blood sampling.

This study protocol involved human subjects, and as such was reviewed and approved by the ethics committees of the University of Medicine and Pharmacy Iuliu Hatieganu Cluj-Napoca (Romania), and the Faculty of Medicine and Pharmacy, Oradea (Romania). The study was conducted according to the principles outlined in the Declaration of Helsinki on experimentation involving human subjects.

\section{Blood and saliva sampling}

Fasting venous blood samples were collected. Blood was processed into serum within $2 \mathrm{~h}$ after collection: whole blood was kept at room temperature for 30-45 min to allow a clot to form, and then centrifuged ( $1500 \mathrm{~g}$ ) for $12 \mathrm{~min}$ to collect the serum. Whole saliva samples were obtained before clinical periodontal measurements and any periodontal intervention by having patients expectorate into polypropylene tubes in the morning following an overnight fast during which subjects were requested not to drink (except water) or chew gum. The saliva samples were centrifuged $(800 \mathrm{~g})$ for $10 \mathrm{~min}$ at $+4^{\circ} \mathrm{C}$. The serum and saliva samples were assayed immediately or frozen at $-40{ }^{\circ} \mathrm{C}$ for biochemical tests and at -20 ${ }^{\circ} \mathrm{C}$ for enzyme-linked immunosorbent assays (ELISAs), and thawed immediately before assays.

\section{Evaluation of renal function}

Serum creatinine (Scr) level was determined according to the manufacturer's instructions. The new guideline recommended using eGFR based on Scr level for initial renal function assessment. eGFR was calculated using the simplified Modification of Diet in Renal Disease (MDRD) formula (eGFR=186 $\times[\mathrm{Scr} / 88,4]^{-1.154} \times[\text { Age }]^{-0.203} \times[0.742$ if Female $\left.]\right)$, where Scr is in micromoles/L, 88.4 is the molecular weight of Scr and age is stated in years. We used the modified National Kidney Foundation classification of CKD, which classifies eGFR into the following ranges: more than $90 \mathrm{~mL} / \mathrm{min} / 1.73$ $\mathrm{m}^{2}$ stage $1 ; 60$ to $89 \mathrm{~mL} / \mathrm{min} / 1.73 \mathrm{~m}^{2}$ stage $2 ; 45$ to $59 \mathrm{~mL} / \mathrm{min} / 1.73 \mathrm{~m}^{2}$ stage $3 \mathrm{a} ; 30$ to $44 \mathrm{~mL} /$ $\min / 1.73 \mathrm{~m}^{2}$ stage $3 \mathrm{~b} ; 15$ to $29 \mathrm{~mL} / \mathrm{min} / 1.73 \mathrm{~m}^{2}$ stage 4 ; and less than $15 \mathrm{~mL} / \mathrm{min} / 1.73 \mathrm{~m}^{2}$ stage 5 (Redal-Baigorri et al., 2013; Levey et al., 2014).

\section{Evaluation of NO synthesis}

NO synthesis was evaluated indirectly by measuring serum levels of nitrites and nitrates 
(NOx). First, serum samples were passed through 10$\mathrm{kDa}$ filters (Sartorius AG, Goettingen, Germany) and contaminant proteins were removed by extraction with a 3:1 (v:v) solution of methanol/diethyl ether. The methanol/diethyl ether ratio in samples was 1:9 (v:v) (Harma et al., 2003). The Griess reaction was used to determine the levels of nitrites and nitrates $\left(\mathrm{NO}_{\mathrm{x}}\right)$ indirectly. In brief, $100 \mu \mathrm{L}$ of $8 \mathrm{mg} / \mathrm{mL} \mathrm{VCl}_{3}$ was added to $100 \mu \mathrm{L}$ of filtered and extracted serum supernatant to reduce nitrates to nitrites, followed by addition of Griess reagents, $50 \mu \mathrm{L}$ of sulfanilamide (2\%) and $50 \mu \mathrm{L}$ of $\mathrm{N}$-(L-naphthyl) ethylenediamine dihydrochloride $(0.1 \%)$. After incubation for $30 \mathrm{~min}$ at $37^{\circ} \mathrm{C}$, absorbance in the sample was read at 540 $\mathrm{nm}$. $\mathrm{NO}_{\mathrm{x}}$ concentration in serum was determined using a sodium nitrite-based curve, and expressed as $\mu \mathrm{mol} / \mathrm{L}$ of nitrite (Miranda et al., 2001).

\section{Evaluation of oxidative stress}

The total oxidative status (TOS) of the serum was measured using a colorimetric assay (Erel, 2005). This assay measured oxidation of the ferrous ion to the ferric ion in the presence of various ROS in an acidic medium. The ferric ion was detected by its reaction with xylenol orange. Assay measurements were standardized using $\mathrm{H}_{2} \mathrm{O}_{2}$ as the oxidative species. Assay results are expressed in $\mu \mathrm{mol} \mathrm{H}_{2} \mathrm{O}_{2}$ equiv/L.

Total antioxidant reactivity (TAR) in the serum was measured using a colorimetric assay (Erel, 2004). This assay measured the rate of production of hydroxyl radicals by the Fenton reaction, which was monitored by following changes in the absorbance of colored dianisidyl radicals. Upon addition of a serum sample, hydroxyl radicalinitiated oxidative reactions were suppressed by antioxidants in the serum. Inhibition of dianisidyl oxidation prevented the subsequent color change, thereby enabling measurement of the total antioxidant capacity of the serum. This assay was calibrated using trolox, and results expressed as mmol trolox equiv/L.

The ratio of TOS to TAR represents oxidative stress index (OSI), an indicator of the degree of oxidative stress (Harma et al., 2003), and is given by the formula:

OSI (arbitrary units) $=\operatorname{TOS}\left(\mu \mathrm{mol} \mathrm{H}_{2} \mathrm{O}_{2}\right.$ equiv/L) $/$ TAR (mmol trolox equiv/L).

\section{Evaluation of MMP-9}

Because circulating levels of MMPs presumably reflect their expression level, serum concen- trations of MMP-9 (gelatinase B) were evaluated by ELISA kits (R\&D Systems, Abingdon, UK). Measurements were performed according to the manufacturer's instructions. Results were calculated by reference to standard curves, and expressed as MMP-9 ng/mL (Musial et al., 2011).

\section{Statistical analyses}

Continuous normally distributed variables were reported as mean and standard deviation (SD). Median and inter-quartile ranges were used to describe non-normally distributed and percentage data. For multiple group comparisons, one-way ANOVA was used, as appropriate. Posthoc analysis was performed using the Tukey or Bonferroni-Holm tests with least significant difference. Variables not normally distributed were logarithmically transformed before being used in parametric comparative analysis. Pearson's and Spearman's correlation analyses were used to calculate relationships between parameters. Significance was attributed when $p<0.05$. All statistical analyses were performed using the Statistical Package for the Social Science, version 16.0 (SPSS Inc., Chicago, IL).

\section{RESULTS AND DISCUSSIONS}

ROS and MMP-9 are produced during periodontal inflammation and tissue destruction. The main finding of this study was that in CP and CKD-CP patients the salivary nitro-oxidative stress markers and MMP-9 concentration were increased and correlated with the clinical periodontal indices. Furthermore, none of the biochemical parameters analyzed in saliva revealed statistically significant differences between the two periodontitis groups. The observation of the present study is preliminary. The results are based on the comparison between the three selected groups, each of 56 individuals. This limits the generalization of the results, which should be verified in a larger population with periodontal diseases.

It is known that individuals have great differences in their rates of disease progression. One objective of periodontal research is to develop diagnostic tests to detect metabolic alterations that occur in the initial phase of periodontal disease (Gonçalves et al., 2009).

Among the 168 eligible participants, clinical diagnoses were made and three mutually exclusive patient groups were categorized. Demographic characteristics of $\mathrm{HC}, \mathrm{CP}$ and CKD-CP patients are 
shown in Table 1. Subjects within groups were generally well matched.

eGFR levels of individuals in the $\mathrm{HC}$ and $\mathrm{CP}$ groups were higher than $90 \mathrm{~mL} / \mathrm{min} / 1.73 \mathrm{~m}^{2}$. In the CKD-CP group, eGFR was significantly lower than in the HC and CP groups $(p<0.0001)$. All CKDCP patients were in CKD stage $3(3 a / 3 b=26 / 30)$ (Table 1). The factors causing CKD were: chronic glomerulonephritis, chronic pyelonephritis, reflux nephropathy, and polycystic kidney disease.

Periodontal clinical measurements showed significantly higher values of PD $(p<0.0001)$, BOP $(p<0.0001)$, and CAL $(p<0.0001)$ in CP and CKDCP groups compared to HC group (Table 1). There were no differences in the periodontal clinical indices $\mathrm{PD}(p=0.19)$ and $\mathrm{BOP}(p=0.10)$ between the $\mathrm{CP}$ and CKD-CP groups. Only the CAL ( $p=0.03)$ score was lower in the CKD-CP group than in the CP group (Table 1).

The use of saliva as sampling material has the advantages of being an easy and noninvasive collection. The wide diversity of biochemical constituents of the human saliva makes it suitable to be used as a biological fluid of diagnosis value. Furthermore, any alternation of biochemical markers of the salivary fluid could be related to a local periodontal disorder or to a systemic disease associated with periodontal disorders. That is why saliva may offer the basis for a patient specific diagnostic test for periodontitis (Gonçalves et al., 2009).

In inflammatory diseases, under conditions of high and sustained NO production, reactive nitrogen oxide species (RNS) are formed through reaction with reactive oxygen species (ROS) (Chapple et al., 2007). NO is produced in vivo from L-arginine by nitric oxide synthase (NOS). The inducible NOS (iNOS/NOS2) is expressed in response to inflammatory stimuli, by various cells. RNS may induce nitrosative and oxidative stress. Nitrosative stress occurs when intermediates are produced from nitrosated thiol, hydroxy and amine groups (Schieber and Chandel, 2014). Oxidative chemistry mediated by RNS is mediated primarily by two nitrogen oxide species, peroxynitrite $\left(\mathrm{ONOO}^{-}\right)$and nitroxyl $\left(\mathrm{NO}^{-}\right)$. Peroxynitrite originates from the reaction between $\mathrm{NO}$ and $\mathrm{O}^{2-}$, while $\mathrm{NO}^{-}$can result from a variety of chemical pathways (Chev et al., 2014).

CKD patients have higher levels of traditional systemic inflammatory markers, like IL-6, and $\mathrm{C}$-reactive protein. Many studies have reported increased prevalence and severity of periodontal disease in patients with CKD (Jain and Kabi, 2013). Free radicals are mostly involved in the destruction of periodontium during various stages of inflammatory periodontal diseases. That is why the nitro-oxidative stress from CKD may be a pathogenetic mechanism for $\mathrm{CP}$ and may become a therapeutic target in those individuals. When CP were compared to $\mathrm{HC}$, a number of alternations have been reported in their salivary biomarkers (Paknjad and Rezaei, 2013). The saliva NOx levels were significantly higher in $\mathrm{CP}(p>0.001)$ and CKD-CP ( $p=0.002)$ patients than in the HC group. Between the CP and CKD-CP groups there was no significant difference $(p>0.05)$ in salivary NO synthesis (Table 2). Compared to the HC group, the CP $(p<0.01)$ and CKD-CP $(p<0.001)$ groups had significantly increased saliva TOS levels (Table 2). CP and CKD-CP had similar effects on saliva TOS production $(p>0.05)$. Salivary NO synthesis was positively correlated to salivary levels of TOS in $\mathrm{CP}(\mathrm{r}=0.74)$ and CKD-CP $(\mathrm{r}=0.91)$ patients. The interesting finding was that $\mathrm{CP}$ did not aggravate the nitro-oxidative stress from the CKD. Salivary

Tab. 1. Demographic characteristics.

\begin{tabular}{lccc}
\hline Parameter & HC & CP & CKD-CP \\
\hline $\mathrm{n}=$ & 56 & 56 & 56 \\
\hline GENDER (M/F) & $18 / 18$ & $16 / 20$ & $19 / 17$ \\
\hline AGE (years) & 43 & 48 & 40 \\
Mean & $30-50$ & $33-68$ & $30-70$ \\
Average & & & \\
\hline
\end{tabular}

Results are given as mean \pm SD; HC, healthy control patients; CP, chronic periodontitis patients; CKD-CP, chronic kidney disease patients with chronic periodontitis. 
cytokines and secretion rates are significantly decreased in CKD (Thorman et al., 2014). That may be an explanation for the fact that there was no significant difference between the nitro-oxidative stress levels in CP and CKD-CP patients.

Antioxidant molecules balance the harmful effects of nitro-oxidative stress. There are enzymatic and non-enzymatic antioxidants. Antioxidant enzymes, including superoxide dismutase and glutathione peroxidase, are less likely to act as antioxidant in extracellular fluid. This is mostly due to their high molecular weights, which do not allow them to pass the membrane without a structural change. It is expected that nonenzymatic antioxidants, that have lower molecular weights, scavenge ROS in the outer cell matrix (Paknjad and Rezaei, 2013). Currently there are no gold standard methods for measuring antioxidant capacity in human (Palmieri and Sblendorio, 2010). Assays of total antioxidant capacity like TAR have been developed in the place of individual antioxidant determination (Erel, 2004).

The systemic antioxidant systems are severely impaired in CP and CKD patients, and worsen progressively with the degree of disease (Ismail et $a l ., 2013)$. The saliva TAR levels were insignificantly changed in both CP and CKD-CP groups ( $p>0.05)$ (Table 2). The saliva OSI values were significantly increased in the CP $(p<0.001)$ and CKD-CP $(p<$ 0.001 ) patients (Table 2 ), and positively correlated with TOS, NOx and the clinical periodontal indices.
That makes TAR a less useful salivary marker for nitro-oxidative stress evaluation in CKD-CP.

Matrix metalloproteinases (MMPs) are zincdependent endopeptidases that participate in the degradation of many components of the extracellular matrix (ECM). They also catalyze the activation reaction of osteoclasts. A special type of this group, MMP-9, is expressed by keratinocytes, fibroblasts, osteoclasts and macrophages. This isozyme is responsible for degrading a variety of the ECM during physiological and pathological conditions (Marcaccini et al., 2009). The severity of periodontitis may be associated with increased metalloproteinase activity.

Higher levels of MMP were found in fluids and gingival tissues from CP patients (Luczyszyn et al., 2012, Marcaccini etal., 2009). The data on systemic MMP-9 concentrations in CKD patients are scarce and contradictory (Gonçalves et al., 2009). Some studies found decreased levels of MMP-9, whereas others noticed no difference between controls and CKD patients (Chang et al., 2006; Tsai et al., 2012; Pawlak et al., 2011). In another study a gradual MMP-9 increase was observed, together with an inverse correlation to GFR values (Musiał and Zwolińska, 2011). Up to now, saliva MMP-9 was not evaluated in CKD-CP patients. In the present study the saliva MMP-9 levels were higher in CP $(p<0.001)$ and CKD-CP $(p<0.001)$ patients compared to the HC patients. There was no significant difference between the salivary MMP-

Tab. 2. Periodontal clinical indices, renal function evaluation, and salivary parameters

\begin{tabular}{lccc}
\hline Parameter & HC & CP & CKD-CP \\
\hline PD $(\mathrm{mm})$ & $1,43 \pm 0,07$ & $6,18 \pm 0,05$ & $5,82 \pm 0,56$ \\
\hline BOP $(\%)$ & $5,1 \pm 0,25$ & $66 \pm 2,91$ & $60,6 \pm 5,98$ \\
\hline CAL $(\mathrm{mm})$ & $1,4 \pm 0,62$ & $7,5 \pm 0,79$ & $6,92 \pm 0,65$ \\
\hline eGRF $\left(\mathrm{mL} / \mathrm{min} / 1.73 \mathrm{~m}^{2}\right)$ & $111,16 \pm 10,54$ & $111,29 \pm 9,83$ & $45,46 \pm 4,87$ \\
\hline NOx $(\mu \mathrm{mole} / \mathrm{L})$ & $25,47 \pm 14,32$ & $48,06 \pm 2,15$ & $44,29 \pm 17,73$ \\
\hline TOS $(\mu \mathrm{mole} \mathrm{H2O2} \mathrm{Equiv./L)}$ & $15,44 \pm 3,05$ & $30,72 \pm 15,50$ & $26,08 \pm 9,86$ \\
\hline TAR $(\mathrm{mmole} \mathrm{TROLOX} \mathrm{Equiv./L)}$ & $1,09 \pm 0,01$ & $1,09 \pm 0,01$ & $1,10 \pm 0,01$ \\
\hline OSI $(\mathrm{AU})$ & $9,75 \pm 2,93$ & $20,82 \pm 9,84$ & $32,21 \pm 6,36$ \\
\hline MMP-9 $(\mathrm{ng} / \mathrm{mL})$ & $56,14 \pm 2,48$ & $67,57 \pm 1,90$ & $64,43 \pm 3,37$ \\
\hline
\end{tabular}

Results are given as mean \pm SD; $\mathrm{HC}$, healthy control patients; $\mathrm{CP}$, chronic periodontitis patients; CKD-CP, chronic kidney disease patients with chronic periodontitis; PD, probing depths; BOP, bleeding on probing; CAL, clinical attachment level; eGFR, estimated glomerular filtration rate. NOx, total nitrites and nitrates; TOS, total oxidative status; TAR, total antioxidant reactivity; OSI, oxidative stress index; MMP-9, matrix metallopeoteinase-9. 
9 levels of CP and CKD-CP patients ( $p>0.5$ ) (Table $2)$. Moreover, MMP-9 changes correlated with the clinical periodontal indices, suggesting that MMP9 plays an important role in tissue injury from $\mathrm{CP}$ as well in CKD-CP.

\section{CONCLUSION}

The change in the salivary parameters analyzed in this study support the hypothesis that saliva nitro-oxidative stress markers and MMP-9 may be used as pathogenetic and prognostic tests in both CP and CKD-CP patients. These findings of significantly increased salivary concentrations of nitro-oxidative stress markers and MMP9 together with significant worsening of the periodontal clinical indices in CP and CKD-CP patients deserve further investigation.

Acknowledgements. This work was financially supported by the Ministry of Education, Research and Youth, CNCSIS grant PNII-IDEI 1273/2008. We would like to thank to Synevo Romania Srl for blood samples collection.

Author Disclosure Statement. The authors state that they have no conflict of interest.

\section{REFERENCES}

1. Anding K, Gross P, Rost JM, Allgaier D, Jacobs E (2003). The influence of uraemia and haemodialysis on neutrophil phagocytosis and antimicrobial killing. Nephrol Dial Transplant 18:2067-2073.

2. Ando M, Shibuya A, Yasuda M, Azuma N, Tsuchiya K, Akiba $\mathrm{T}$, et al. (2005). Impairment of innate cellular response to in vitro stimuli in patients on continuous ambulatory peritoneal dialysis. Nephrol Dial Transplant 20:24972503.

3. Ardalan MR, Ghabili K, Pourabbas R, Shoja MM (2011). A causative link between periodontal disease and glomerulonephritis: a preliminary study. Ther Clin Risk Manag 7:93-98.

4. Carracedo J, Merino A, Nogueras S, Carretero D, Berdud I, Ramirez R, et al (2006). On-line hemodiafiltration reduces the proinflammatory CD14+CD16+ monocyte-derived dendritic cells: a prospective, crossover study. J Am Soc Nephrol 17:2315-2321.

5. Chang HR, Yang SF, Li ML, Lin CC, Hsieh YS, Lian JD (2006). Relationships between circulating matrix metalloproteinase- 2 and -9 and renal function in patients with chronic kidney disease. Clin Chim Acta 366(1-2): 243-248.

6. Chapple ILC, Matthews JB (2007). The role of reactive oxygen and antioxidant species in periodontal tissue destruction, Periodontology 2000 43: 160-232.
7. Chen X, Chen H, Deng R, Shen J (2014). Pros and cons of current approaches for detecting peroxynitrite and their applications. Biomed J 37(3):120-126.

8. CraigRG,KotankoP,KamerAR, Levin NW(2007).Periodontal diseases-a modifiable source of systemic inflammation for the endstage renal disease patient on haemodialysis therapy? Nephrol Dial Transplant 22(2):312-315.

9. Dağ A, Firat ET, Kadiroğlu AK, Kale E, Yilmaz ME (2010). Significance of elevated gingival crevicular fluid tumor necrosis factor-alpha and interleukin-8 levels in chronic hemodialysis patients with periodontal disease. J Periodontal Res 45(4):445-450.

10. Erel 0 (2004). A novel automated method to measure total antioxidant response against potent free radical reactions. Clin Biochem 37: 112-119.

11. Erel $O$ (2005). A new automated colorimetric method for measuring total oxidant status. Clin Biochem 38: 11031111.

12. Gonçalves RP, Damante CA, Lima FLM, Imbronito AV, Nunes FD, Pustiglioni FE (2009). Detection of MMP2 and MMP-9 salivary levels in patients with chronic periodontitis before and after periodontal treatment. Rev. odonto ciênc 24(3):264-269.

13. Graziani F, Cei S, La Ferla F, Vano M, Gabriele M, Tonetti $M$ (2010). Effects of non-surgical periodontal therapy on glomerular filtration rate of the kidney: an exploratory trial. J Clin Periodontol 37: 638-643.

14. Grubbs V, Plantinga LC, Crews DC, Bibbins-Domingo K, Saran R, Heung M, et al. (2011). Vulnerable populations and the association between periodontal and chronic kidney disease. Clin J Am Soc Nephrol 6(4):711-717.

15. Harma M, Harma M, Erel O (2003). Increased oxidative stress in patients with hydatidiform mole. Swiss Med Wkly 133: 563-566.

16. Huang R-Y, Lin Y-F, Kao S-Y, Shieh Y-S, Chen J-S (2014). A Retrospective Case-Control Analysis of the Outpatient Expenditures for Western Medicine and Dental Treatment Modalities in CKD Patients in Taiwan. PLoS ONE 9(2): e88418.

17. Ioannidou E, Hall Y, Swede H, Himmelfarb J (2013). Periodontitis associated with chronic kidney disease among Mexican Americans. J Public Health Dent. 73(2):112-129.

18. Ioannidou E, Swede H, Dongari-Bagtzoglou A (2011). Periodontitis predicts elevated C-reactive protein levels in chronic kidney disease. J Dent Res 90(12):1411-1415.

19. Ioannidou E, Swede H, Fares G, Himmelfarb J (2014). Tooth loss strongly associates with malnutrition in chronic kidney disease. J Periodontol 85(7):899-907.

20. Ioannidou E, Swede H (2011). Disparities in periodontitis prevalence among chronic kidney disease patients. J Dent Res 90(6):730-734.

21. Ismail G, Dumitriu HT, Dumitriu AS, Ismail FB (2013). Periodontal Disease: A Covert Source of Inflammation in Chronic Kidney Disease Patients. Int J Nephrol Article ID 515796. 
22. Jain A, Kabi D (2013). Severe periodontitis associated with chronic kidney disease. J Indian Soc Periodontol 17:128-130.

23. Kato S, Chmielewski M, Honda H, Pecoits-Filho R, Matsuo S, Yuzawa Y, et al. (2008). Aspects of immune dysfunction in end-stage renal disease. Clin J Am Soc Nephrol 3:15261533.

24. Levey AS, Inker LA, Coresh J (2014). GFR estimation: from physiology to public health. Am J Kidney Dis 63(5): 820834.

25. Luczyszyn SM, de Souza CM, Braosi AP, Dirschnabel AJ, Claudino M, Repeke CE, et al. (2012). Analysis of the association of an MMP1 promoter polymorphism and transcript levels with chronic periodontitis and end-stage renal disease in a Brazilian population. Arch Oral Biol 57(7):954-963.

26. Machida T, Tomofuji T, Ekuni D, Yamane M, Yoneda T, Kawabata Y, et al. (2014). Longitudinal relationship between plasma reactive oxygen metabolites and periodontal condition in the maintenance phase of periodontal treatment. Dis Markers 489292

27. Marcaccini AM, Novaes AB Jr, Meschiari CA, Souza SL, Palioto DB, Sorgi CA, et al. (2009). Circulating matrix metalloproteinase-8 (MMP-8) and MMP-9 are increased in chronic periodontal disease and decrease after nonsurgical periodontal therapy. Clin Chim Acta 409(12):117-122.

28. Miranda KM, Espey MG, Wink DA (2001). A rapid, simple spectrophotometric method for simultaneous detection of nitrate and nitrite. Nitric Oxide 5: 62-71.

29. Musiał K, Zwolińska D (2011). Matrix metalloproteinases (MMP-2,9) and their tissue inhibitors (TIMP-1,2) as novel markers of stress response and atherogenesis in children with chronic kidney disease (CKD) on conservative treatment. Cell Stress Chaperones 16(1):97-103.

30. Offenbacher S (1996). Periodontal diseases: pathogenesis. Ann Periodontol 1:821-878.

31. Paknjad M, Rezaei A (2013). Salivary biochemical markers of periodontitis. Rom J Biochem 50(2):129-146.

32. Palmieri B, Sblendorio V (2010). Current status of measuring oxidative stress. Methods Mol Biol 594: 3-17.

33. Pârvu AE, Alb SF, Crăciun A, Taulescu MA (2013). Efficacy of subantimicrobial-dose doxycycline against nitrosative stress in chronic periodontitis. Acta Pharmacol Sin 34(2): 247-254.

34. Pârvu AE, Tălu S, Crăciun C, Alb SF (2014). Evaluation of scaling and root planing effect in generalized chronic periodontitis by fractal and multifractal analysis. J Period Res 49(2):186-196.

35. Pawlak K, Mysliwiec M, Pawlak D (2011). Peripheral blood level alterations of MMP-2 and MMP-9 in patients with chronic kidney disease on conservative treatment and on hemodialysis. Clin Biochem 44(10-11):838-843.

36. Pizzo G, Guiglia R, Lo Russo L, Campisi G (2010). Dentistry and internal medicine: from the focal infection theory to the periodontal medicine concept. Eur J Intern Med 21(6):496-502.

37. Pradeep AR, Kathariya R, Arjun Raju P, Sushma Rani R, Sharma A, Raghavendra NM (2012). Risk factors for chronic kidney diseases may include periodontal diseases, as estimated by the correlations of plasma pentraxin-3 levels: a case-control study. Int Urol Nephrol 44(3):829839.

38. Redal-Baigorri B, Rasmussen K, Heaf JG (2013). The use of absolute values improves performance of estimation formulae: a retrospective cross sectional study. BMC Nephrol 14: 271.

39. Roberts RA, Laskin DL, Smith CV, Robertson FM, Allen EM, Doorn JA, Slikker W (2009). Nitrative and oxidative stress in toxicology and disease. Toxicol Sci 112(1):4-16.

40. Roberts RA, Smith RA, Safe S, Szabo C, Tjalkens RB, Robertson FM (2010). Toxicological and pathophysiological roles of reactive oxygen and nitrogen species. Toxicology 276(2):85-94.

41. Schieber M, Chandel NS (2014). ROS Function in Redox Signaling and Oxidative Stress. Curr Biol 24(10): R453-R462.

42. Shaqman M, Ioannidou E, Burleson J, Hull D, DongariBagtzoglou A (2010). Periodontitis and inflammatory markers in transplant recipients. J Periodontol 81(5):666672 .

43. Thorman R, Lundahl J, Yucel-Lindberg T, Hylander B (2010). Inflammatory cytokines in saliva: early signs of metabolic disorders in chronic kidney disease. A controlled cross-sectional study. Oral Surg Oral Med Oral Pathol Oral Radiol Endod 110(5):597-604.

44. Tomofuji T, Ekuni D, Irie K, Azuma T, Tamaki N, Maruyama T, Yamamoto T, Watanabe T, Morita M (2011) Relationships between periodontal inflammation, lipid peroxide and oxidative damage of multiple organs in rats. Biomed Res 32(5):343-349.

45. Tsai J-P, Liou JH, Kao WT, Wang SC, Lian JD, Chang HR (2012). Increased Expression of Intranuclear Matrix Metalloproteinase 9 in Atrophic Renal Tubules Is Associated with Renal Fibrosis. PLoS ONE 7(10): e48164.

46. Wan C, Yuan G, Yang J, Sun Q, Zhang L, Zhang J, et al. (2014). MMP9 deficiency increased the size of experimentally induced apical periodontitis. J Endod 40(5):658-664.

47. Wehmeyer MM, Kshirsagar AV, Barros SP, Beck JD, Moss KL, Preisser JS, et al. (2013). A randomized controlled trial of intensive periodontal therapy on metabolic and inflammatory markers in patients With ESRD: results of an exploratory study. Am J Kidney Dis 61(3):450-458. 